\title{
A bibliometric analysis of micro/nano-bubble related research: current trends, present application, and future prospects
}

\author{
Tianlong Zheng ${ }^{1,2} \cdot$ Juan Wang ${ }^{1}$ Qunhui Wang ${ }^{1,3}$.

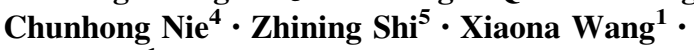 \\ Zhen Gao ${ }^{1}$
}

Received: 15 November 2015/Published online: 3 June 2016

(C) Akadémiai Kiadó, Budapest, Hungary 2016

\begin{abstract}
With great versatile characteristics, micro/nano-bubble related research have attracted much attention due to their extensive applications in the last half century. Researchers not merely focus on their physi-chemical properties, but also aim at their wellcontrolled generation methods and potential adhibition field. It can be expected that the future prospects of micro/nano-bubble related research will be tremendous and that there will be even more to be explored. In this case study, a bibliometric analysis was conducted to evaluate micro/nano-bubble related research from 1991 to 2014, based on the Science Citation Index EXPANDED database. The Ultrasound in Medicine and Biology with the highest $h$-index of 56 is the leading journal in this field, publishing $6.9 \%$ of articles over this period, followed by Langmuir and Journal of the Acoustical Society of America. USA and the Univ Toronto, Canada were the most productive country and institution, respectively, while the USA, was the most internationally collaborative and had the highest $h$ index (111) of all countries. A new method named "word cluster analysis" was successfully applied to trace the research hotspots. Innovation in detection means and novel pathways for medical applications via micro/nano-bubble is considered to relate to the
\end{abstract}

Electronic supplementary material The online version of this article (doi:10.1007/s11192-016-2004-4) contains supplementary material, which is available to authorized users.

Qunhui Wang

wangqh59@sina.com

1 Department of Environmental Engineering, University of Science and Technology Beijing, 30 Xueyuan Road, Haidian District, Beijing 100083, China

2 Research Center for Eco-Environmental Sciences, Chinese Academy of Sciences, 18 Shuangqing Road, Haidian District, Beijing 100085, China

3 Beijing Key Laboratory of Resource-Oriented Treatment of Industrial Pollutants, University of Science and Technology Beijing, 30 Xueyuan Road, Haidian District, Beijing 100083, China

4 Investment and Development Department, Jiangxi Tungsten Industry Group Co., Ltd, 188 Huoju Street, Nanchang 330096, Jiangxi, China

5 School of Earth and Environmental Sciences, The University of Adelaide, Adelaide, SA 5005, Australia 
increasingly new types and varieties of diseases or cancers, as well as the well-controlled generation of micro/nano-bubbles.

Keywords Bibliometric analysis - Case study - Micro/nano-bubble $\cdot$ Research trends Scientometrics · SCI-EXPANDED

\section{Introduction}

Microbubbles with diameters ranging from a few micrometers to tens of micrometers have drawn significant attention (Zheng et al. 2015d). The diameters of nanobubbles with less than $200 \mathrm{~nm}$ are far removed from these of microbubbles (Agarwal et al. 2011). When Sebba (1971) first reported that tiny bubbles with diameters of several tens of micrometers differ from their larger counterparts in many important properties, with distinctive characteristics including huge interfacial area, long stagnation time, lower bubble rising speed, and high interior pressure which are different from ordinary bubbles (Zheng et al. 2015c), micro/nano-bubble had been widely used in various medical (Lee et al. 2015), environmental pollution control (Xu et al. 2011), food processing (Sato 2011), marine (Endo et al. 2008) and agriculture (Neethirajan et al. 2011) applications. In this current study, the global research trends of micro/nano-bubbles are introduced in detail mainly on generation methods, medical applications, and future prospects, which not only focus on the technique of micro/nano-bubbles used in some specific scientific areas but also for the phenomenon of micro/nano-bubbles that is presented in some investigations.

A variety of techniques have been extensively developed to generate the micro/nanobubbles, such as mechanical agitation (Xu et al. 2008), sonication (Xu et al. 2008), ultrasonication (Lee et al. 2015), and induced air flotation (Li and Tsuge 2006). The mechanical agitation technique (Xu et al. 2008) could be defined as the first generation for the production of microbubbles with a diameter smaller than $100 \mu \mathrm{m}$, which the microbubble experienced a slow reduction in diameter within the observation period (120 s). Due to the bigger critical diameter and longer processing time, the other novel kinds of microbubbles generation methods was investigated. The microbubbles generated by sonication (Xu et al. 2008) had a diameter smaller than $50 \mu \mathrm{m}$ and a shrinkage was observed. Moreover, its shrinkage rate increased with the decrease of the size of microbubbles. In order to explore a promising fabrication route for large-scale generation of microbubbles with lower cost, stochastic approach of ultrasonication (Lee et al. 2015) was applied to generate turbulent flows, which was in favor of formation of stable bubbles. Whereas, the tunability of the bubble size and distribution was inevitably sacrificed, which seems adverse to its practical application. The other technique of induced air flotation ( $\mathrm{Li}$ and Tsuge 2006) for microbubble generation was always considered as an alternative to the dissolved air flotation in dealing with wastewater queries. Furthermore, the micro/nano-bubbles could also be generated under some specific phenomenon with or without other auxiliary technologies (Sassaroli and Hynynen 2007; Neethirajan et al. 2011). Insonated by specific ultrasound pulse, the cavitation phenomenon (Sassaroli and Hynynen 2007) could be used to produce the microbubbles, which also offer a novel chance to study the microbubble collapse. In comparison to ultrasonication technique, the microfluidics approach (Neethirajan et al. 2011) performed an opposite extreme and capacitated the generation of microbubbles with high uniformity which can be readily controlled by the liquid flow rate, the physichemical properties of the fluids, and the microfluidic devices (Lee et al. 2015). 
With a rapidly growing body of research in the new era, the introduction of micro/nanobubbles had been extensively improved the development of the healthcare industry (Unnikrishnan and Klibanov 2012). For instance, micro/nano-bubbles have become the most effective type of ultrasound contrast agent available for ultrasound imaging, personalized diagnostics, and molecular imaging, and they are widely used in other therapeutic applications, such as ultrasonography, sonoporation, echocardiography, angiogenesis and thrombolysis, and as efficient carriers of gas, genes and drugs for therapeutic and clinical applications (Aoi et al. 2008; Gao et al. 2008; Delalande et al. 2013; Unger et al. 2014). However, there are still significant problems to be solved, such as the accurately controlled stability of bubbles, dimensions of bubbles in desired ranges, and bubbling frequency (Rodríguez-Rodríguez et al. 2015). Therefore, how to develop practical micro/nano-bubble generation with low-cost,energy efficiency,superior performance and easy application in this broad field is a promising but challenging area of research.

Bibliometrics, which gained insight into the development trends of various research fields through a quantitative analysis and statistics, firstly introduced by Pritchard (1969). Science Citation Index EXPANDED (SCI EXPANDED) was the most important and frequently used source database for an overview of scientific production. Bibliometric methods have been used to assess the scientific outputs of nano-technology field, such as global nanobiotechnology research (Karpagam 2014), carbon nanostructures (Terekhov 2015), nanofiltration membrane technology (Zhai et al. 2014), and nanotechnology for social needs: contributions from Latin American research in the areas of health, energy and water (Invernizzi et al. 2015). In addition, research patterns including journals, categories, countries, and institutes, as well as the international cooperation had been broadly applied (Mao et al. 2015; Wang et al. 2015; Zheng et al. 2015a). Moreover, h-index, namely the number $\mathrm{h}$ of publications with at least h citations, which was introduced by Hirsch (2005), had become a valid indicator on scientific achievement (Hirsch 2007; Ciriminna and Pagliaro 2013). In addition, the analysis of author keywords in different periods also had been used to evaluate the research trend in recent years (Wang et al. 2013; Fu et al. 2014).

In this study, a comprehensive bibliometric analysis was used to trace global trends in micro/nano-bubble related research from 1991 to 2014. Document types, languages, categories, journals, countries/territories, institutions, and h-index were analyzed to dissect publication patterns. Further, research tendencies and hotspots including the distribution of author keywords in different periods and 'word cluster analysis' (Mao et al. 2010) were also applied. Our results provide a basis for the comprehensive understanding of micro/nano-bubble related research, and serve as a potential guide for future directions.

\section{Data sources and methodology}

\section{Data sources}

Information about scientific output was extracted from the SCI-EXPANDED database and the 2014 Journal Citation Report (JCR) of Thomson Reuters, on the 29th of June, 2015. The 2014 JCR indexes 11,149 journals across 237 scientific disciplines and 82 countries. Since 1991, abstract information has been included in ISI Web of Science. In this study, [microbubble* or nanobubble* or (micro or nano)-bubble* or ((micro or nano) bubble*)] was used as a topic search phrase to search topics in SCI-EXPANED for the period from 1991 to 2014. 


\section{Data analysis}

Document type, language, output, subject category, journal, country, institute, source title, abstract keyword, and h-index were all analyzed by Microsoft Excel 2010. Frequency analysis was conducted using BibExcel 1.0.0.0 (Persson et al. 2009). Data downloaded from Web of Science can be read by BibExcel and then be used for co-occurrence analysis and frequency analysis. Network diagrams for cooperation analysis were generated by Pajek 1.0.0.1 (De Nooy et al. 2011). The contribution of different countries and institutes were estimated by the location of the affiliation of at least one author of the published papers. Articles that originated from Hong Kong were included under China. Articles that originated from England, Scotland, Northern Ireland, and Wales were grouped under the UK. "Internationally collaborative publication" was defined as being articles that were authored by researchers from more than one country, whereas "independent type" was assigned if the researchers' addresses were from the same country. "Single institute publication" was assigned if the researchers' addresses were from the same institute. The term "inter-institutionally collaborative publication" was assigned if the authors were from different institutes. $h$-index was defined by the $\mathrm{h}$ of $\mathrm{Np}$ papers having at least $h$ citations each and the other $(\mathrm{Np}-h)$ papers having $\leq h$ citations each. The reported journal impact factor (JIF) was obtained from the JCR Science Edition 2014. The new generation of the InCites platform provides enhanced JIF Quartile calculation, with a greater level of precision (Thomson Reuters 2015), so where quartile data differed between JCR on the Web of Science platform and JCR on the InCites platform, the latter record was used.

\section{Results and discussion}

\section{Publication pattern}

\section{Document type and language of publication}

9444 publications related to the micro/nano-bubble research were identified in SCIEXPANDED between 1991 and 2014, which were categorized by 12 document types. Articles were the dominant document type comprising $78.4 \%$ or 7404 of the total. The remaining publications were proceedings (690), meeting abstract (631), review (528), editorial material (97), letter (38), news item (17), note (16), correction (13), book chapter (7), addition (2), and reprint (1). On average, there were 11 articles per proceedings and 12 articles per meeting abstract.

As articles were the dominant type of publications, other types were discarded from further analysis. 7248 articles (98\%) were published in English, followed by German (52), Chinese (36), French (30), Japanese (20), Spanish (4), Portuguese (3), Russian (2), Polish (2), Korean (2), and only one article each in Czech, Italian, Ukrainian, Rumanian, and Lithuanian, respectively.

\section{Characteristics of publication outputs}

As there are no abstracts in SCI-EXPANDED prior to 1991, [Microbubble* OR nanobubble* OR (micro or nano)-bubble* OR ((micro or nano) bubble*)] was used as a topic search phrase for topics to obtain a general long-term trend. The earliest research 
indexed within SCI-EXPANDED on micro/nano-bubble related research was published in 1949. Figure 1 shows that the amount of micro/nano-bubble related research slowly and continually grew from 1945 to 1990 . The number of publications rose significantly from 1991.

The number of articles published per year grew from 46 in 1991 to 815 in 2014. An exponential model describes the cumulative annual number of articles from 1991 (Fig. 2), with the equation $C=-437.28+347.04 e^{0.13 Y}\left(R^{2}>0.999\right)$, where $C$ is the cumulative number of articles and $Y$ is the number of years since 1991. Based on this exponential model, the cumulative number of articles to be published from 1991 to 2015 is 8641 . The number of articles published in 2019 (1873) is predicted to be twice that of 2014 (815).

Appendix Table 1 of online supplement shows that the mean number of authors per article increased by $39.5 \%$ from 3.8 in 1991 to 5.3 in 2014, with a similar 33.2 and $33.3 \%$ increase in the number of pages and institutions per article respectively over the same period. The mean number of references cited per article increased as high as $73.4 \%$, from 21.5 in 1991 to 37.4 in 2014 . The average number of countries per article was stable at 1.2 countries. The number of times each article was cited firstly fluctuated slightly, then decreased with a peak average of 52.1 citations in 1996. The most frequent number of authors was four, accounting for 1232 articles (16.6\%). Two to six authors accounted for 5279 articles $(71.3 \%)$ and 282 articles $(3.8 \%)$ had one author. The maximum number of authors per article was 27 [which was published on European Radiology with a JIF (4.014) and $h$-index (23) named "Liver fibrosis staging with contrast-enhanced ultrasonography: prospective multicenter study compared with METAVIR scoring" (Staub et al. 2009)]. The 7404 articles contained a total of 63,861 pages, with most having between six and eight pages six to eight pages $(2905 ; 39.2 \%)$.

Of the 7404 articles, the most frequently cited articles had each been cited more than 950 times by 2014 . The next most frequently cited articles followed distantly with less than 700 citations. The most frequently cited research article, with 986 citations, as well as the 2rd most frequently cited research article per year with 58, was 'Quantification of myocardial blood flow with ultrasound-induced destruction of microbubbles administered as a constant venous infusion' (Wei et al. 1998) published in Circulation from Univ

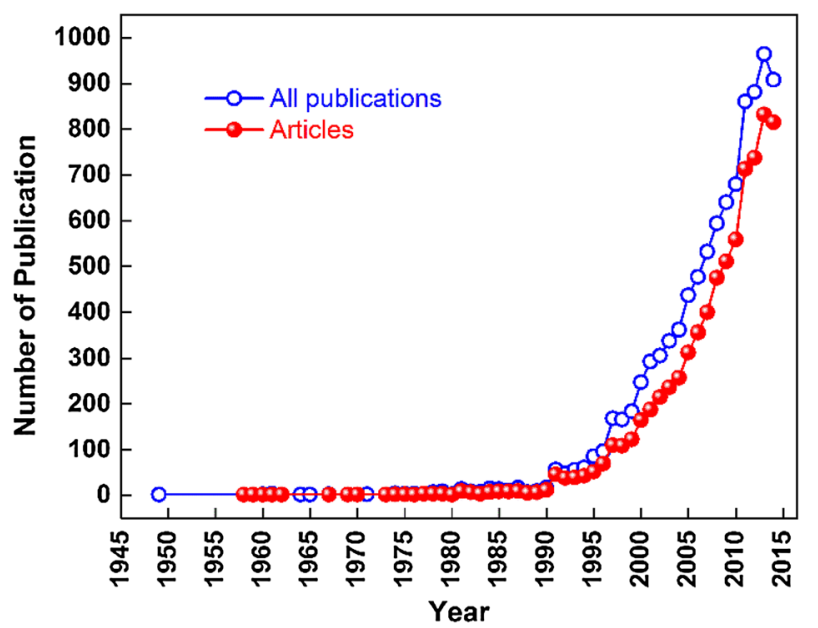

Fig. 1 Number of SCI publications on micro/nano-bubble related research over last 70 years 


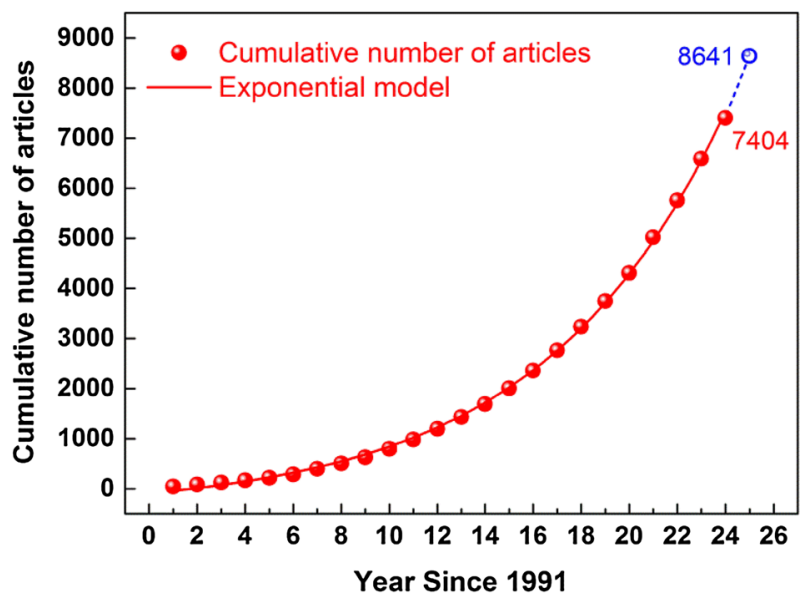

Fig. 2 The relationship between cumulative number of publications and years since 1991

Virginia, USA, which derived a new approach with myocardial contrast echocardiography during a venous infusion of microbubbles to quantify myocardial blood flow. The most frequently cited research article per year with 79 named 'strain-induced pseudo-magnetic fields greater than $300 \mathrm{~T}$ in graphene nanobubbles' was published in Science from Univ Calif Berkeley, USA (Levy et al. 2010).

\section{Output in subject categories and journals}

The 7404 articles were published in 1476 journals in 103 subject categories in SCIEXPANDED. Among these journals, 1344 (91\%) journals contained less than 10 articles. The top 20 most productive journals (TP $>55$ ) with ISI category, position of the journal within its category, JIF in 2014, h-index and journal country are summarized (see in Appendix Table 2 of online supplement). These top 20 most productive journals accounted for appropriately $30 \%$ of the total articles.

Ultrasound in Medicine and Biology with the highest $h$-index (56), published the most articles $(510 ; 6.9 \%)$, followed by Langmuir (166; $2.2 \%)$, Journal of the Acoustical Society of America (152; $2.1 \%)$, and IEEE Transactions on Ultrasonics Ferroelectrics and Frequency Control (142; $1.9 \%$ ). Interestingly, Circulation (57; $0.8 \%)$, with the highest JIF (14.43) and $2^{\text {nd }}$ highest $h$-index (43) in 2014, ranked top in the category of peripheral vascular disease (Q1: 1/60), though ranked 18th for overall productivity. It was worth mentioning that the relationship between $h$-index, JIF and the rank order of the 20 most productive journals revealed extremely different trends. For example, Journal of Ultrasound in Medicine was 5th in the top 20 most productive journals yet with JIF (1.535) and h-index (27) ranked 17th and 9th, respectively; on the contrary, Journal of Controlled Release ranked 10th in the top 20 of most productive journals, while JIF (7.705) and $h$ index (32) ranked 2nd and 5th. This may be attributable to the highest impact journals being both less specialized, and more selective of research quality and novelty. Among the top 20 journals, twelve journals are from the USA, five from the UK, two from the Netherlands, one from the Germany, which shows dominant status of developed countries in journal publication. Due to the assignment of journals to multiple subject categories, 
micro/nano-bubble related research covered $103 \mathrm{Web}$ of Science categories. As illustrated in Fig. 3, based on the continuous increase in the number of articles per category, micro/nano-bubble related research expressed similar trends to those that have been seen in many fields of science since the beginning of the 21st century. The first echelon of most common subject categories were 'Radiology, Nuclear Medicine \& Medical Imaging', 'Engineering', and 'Acoustics', which kept good momentum during the research period. The other three popular subject categories belonging to the second echelon were 'Chemistry', 'Physics', and 'Materials Science', which rapidly increased during the last decade. In addition, the research trends in these fields are hotspots identified in the present study.

Of the 1476 journals that published the 7404 articles, 735 (49.8\%) journals contained 1 article, $247(16.7 \%)$ journals contained 2 articles, 109 (7.4\%) journals contained 3 articles and $72(4.9 \%)$ journals contained 4 articles. Approximately 1187 (16.0\%) of the articles were published in 6 core journals. Publication trends for the 6 journals with the greatest number of articles are shown in Fig. 4.

\section{Publication performances: institutes and countries}

The contribution of different countries and territories were estimated by the affiliation of at least one author of the publications. 7392 articles with author address information published between 1991 and 2014 were analyzed, covering 79 countries/territories. 5952 $(80.5 \%)$ were independent articles and $1440(19.5 \%)$ were internationally collaborative articles. The top 25 most productive countries for total articles. Total number of single country and internationally collaborative articles, the percentage of single country articles of total articles, the percentage of internationally collaborative articles of total articles, and the $h$-index of countries/territories are analyzed (see in Appendix Table 3 of online supplement). Thirteen European countries, eight Asian countries/territories, three American countries, and one Oceanic country were ranked in the top 25 productive countries of articles. The top 25 most productive countries are responsible for $94.7 \%$ of all articles. The seven major industrialized countries (G7: Canada, France, Germany, Italy, Japan, the UK, and USA), ranked in the top 8, producing $59.4 \%$ of all articles over the investigative period. Developed countries hold a dominant position in micro/nano-bubble related research, demonstrating the economic capabilities and academic levels of these countries (Bell and Pavitt 1997). Of the BRICS countries (Finardi 2015), India, China, Brazil, and

(a)

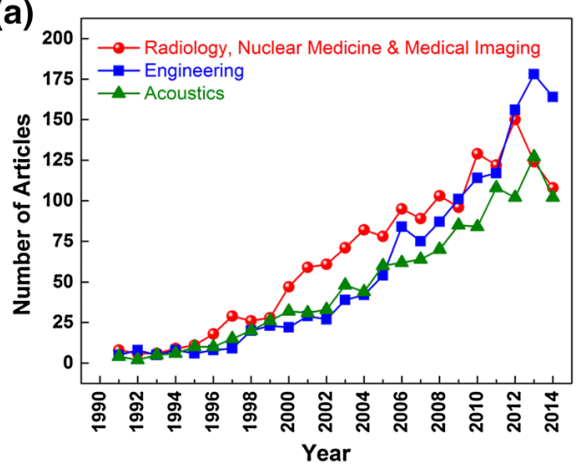

(b)

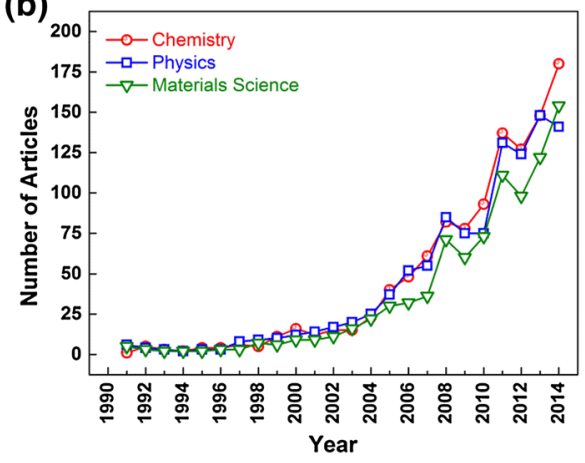

Fig. 3 The growth trends of the top six subject categories 

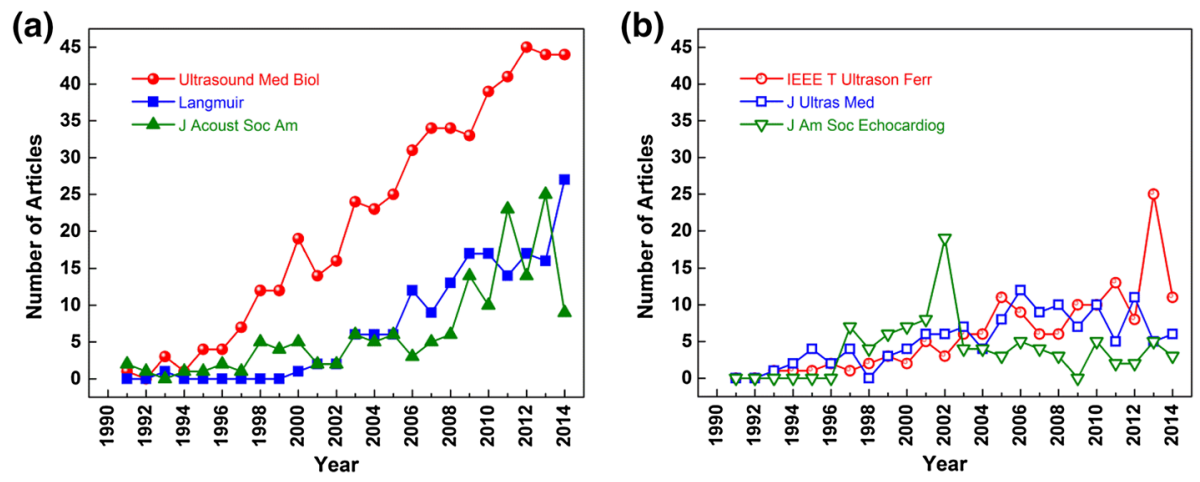

Fig. 4 The growth trends of the top six journals

Russia were found to be productive with 1423 (15.6\%) of the total articles. Besides, South Africa ranked 48th among all 80 countries/territories.

USA ranked first in total articles (2344), independent articles (1674), collaborated articles (670), first authored articles (1969) and corresponding authored articles (1912), and had the highest h-index (111). Followed USA, China occupied 2nd during all above described fields, however, China was ranked 20th of the 25 countries on percentage of collaboration with outside authors, while Switzerland ranked 13th in the number of articles yet had the highest proportion of collaborative articles. The proportions of internationally collaborative articles to the total outputs of these 25 countries showed significant disparity. Yet, the USA with the highest $h$-index of 111 not only contributed the most collaborative articles (670), but was also the major collaborator of 20 other countries (see in Appendix Table 3 of online supplement), indicating that the USA still undoubtedly had leadership in this field.

Further, although single-country articles still dominate in the micro/nano-bubble related research, international collaboration is becoming increasingly popular, which is a trend shared with other fields (Niu et al. 2014). The academic collaborative relationships among the top 25 most productive countries were chosen as study samples, which were firstly grouped into different clusters in BibExcel with an algorithm developed by Persson (1994) and then graphed using Pajek (Fig. 5). In this figure, each circle represents a country. The size of the circle is proportional to the total number of articles produced by the country and the line weight and darkness are proportional to the extent of cooperation, with respect to papers produced by authors from the two countries.

Figure 5 shows that showed that the USA, in a core position in the collaboration network, cooperated frequently with China, Germany, Canada, UK, Japan, France, Italy, and Netherlands, with 153, 72, 67, 54, 53, 43, 41, and 40 cooperative publications, respectively. Besides, Germany showed a close collaborative relationship with other European countries such as Switzerland, UK, France, Italy, and Netherlands. Similarly, the China-Japan collaborations was outstanding with 36 joint publications. Collaborative countries tend to be geographically correlated and centre into the countries ranking higher in terms of total publications, which is mirrored by findings in other scientific fields (Niu et al. 2014; Gao et al. 2015; Mao et al. 2015).

The USA and China are the top two productive countries from 1991 to 2014, making up 31.7 and $15.3 \%$ of global articles, distantly followed by next most productive countries 


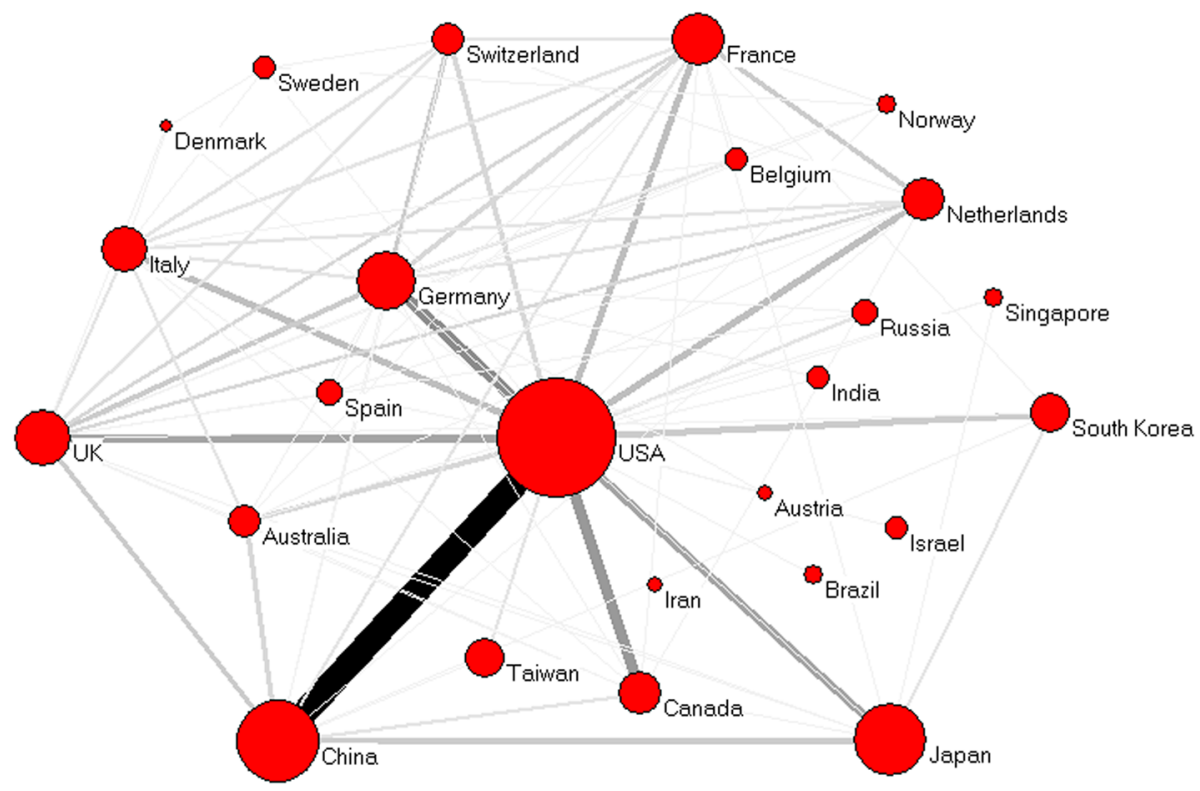

Fig. 5 Network diagram showing cooperation between the top 25 most productive countries with a minimum of five articles

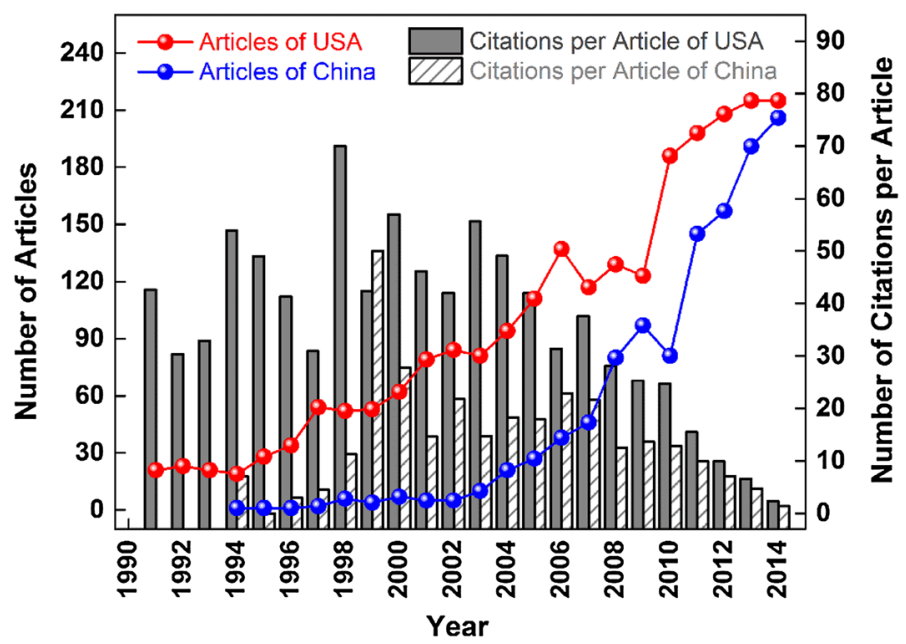

Fig. 6 The growth trends of articles and citations per year in USA and China from 1991 to 2014

with less than 900 articles. The growth trends of articles and citations per year of USA and China were investigated (Fig. 6).

Figure 6 shows that the USA continuously ranked 1st in number of articles per year during all the research period. In addition, the USA has a much longer history of research in this field than China. The USA published its first paper in 1978 and it was 15 years later that China published its first paper. However, China has undergone rapid growth in the 
number of articles produced over the last decade and possibly transcends USA in the year of 2015, which can be attributed to both increasing investment in scientific research and domestic concern for medical environment issues (Zhou and Leydesdorff 2006; Yu et al. 2011). Figure 6 also shows that a major gap existed between China and the USA in terms of total number of citations for all articles per year from 1991 to 2006, excluding one outlier. The outlier value was caused by two highly cited articles in only four articles samples in 1999, one of which comprises of $50 \%$ of the total citations (Xu et al. 1999) and the other with $45 \%$ of the total citations (Jiang et al. 1999). Though all these studies concluded the USA was still leading the China in numbers and quality of these publications, the China was rapidly closing this gap in the last decade. The progress China has made as a dominant producer of research in this field implies positive policy decisions by the Chinese government.

At the institution level, 7392 articles came from 4108 institutes in 79 countries. 4005 $(54.2 \%)$ were inter-institutionally collaborative publications, and 3387 (45.8\%) were independent publications. The percentage of collaboration between institutes was $34.7 \%$ higher than that between countries. Eight institutes in the USA, five in China, two each in Canada, UK, Taiwan, and Netherlands, and one each in Australia, Russia, France, and Japan, were ranked in the top 25 most productive institutes (see in Appendix Table 4 of online supplement).

The Univ Toronto, Canada with an h-index of 29 (6th) was the most productive institute for total articles (156), as well as for inter-institution collaborative articles (139). Both Chinese Acad Sci, China and Univ Virginia, USA with 154 articles (2nd) are not far behind. There is a bias for the Chinese Academy of Sciences as it has branches in many cities. In this study, articles produced by this institute were regarded as being under one heading; dividing the articles into branches would make a difference in ranking (Zheng et al. 2015b). Interestingly, The Univ Virginia, USA, with a highest $h$-index of 58 and 2nd highest output, ranked 23rd of 25 in inter-institution collaborative articles; on the contrary, the Erasmus MC, Netherlands, ranked 10th in the most productive institutes ranked 1st $(93.75 \%)$ in the number of inter-institutionally collaborative articles. In addition, $69.2 \%$ of articles produced by the 25 most productive institutions in micro/nano-bubble related articles were inter-institutionally collaborative. It is obvious that institutions from the same country tended to have higher rates of collaboration, and the major collaborative institutions of these 20 institutions were almost all (24 of 25) from the same country.

It is apparent that collaboration plays an ever-growing role in the micro/nano-bubble literature. Among the 7392 articles with address information, 3387 (45.8\%) were independent articles published by a single institution, with the rest inter-institutional collaborative works, including both national (34.7\%) and international $(19.5 \%)$ collaborations. As seen in Fig. 7, the number of collaborative articles exceeded the number of independent articles in 2007 and ultimately accounted for $59.9 \%$ of total articles in 2014. In general, the increasing trend of collaborative articles relative to the total can be partly explained by the fact that the number of institutes and countries engaged in the micro/nano-bubble related research increased. The dominant form of collaborative article was national collaboration $(67.5 \%)$, in which collaborators were located in the same country. The proportion of internationally collaborative articles has gradually increased, from $21.1 \%$ of all collaborative articles in 1991 to $35.5 \%$ in 2014. 


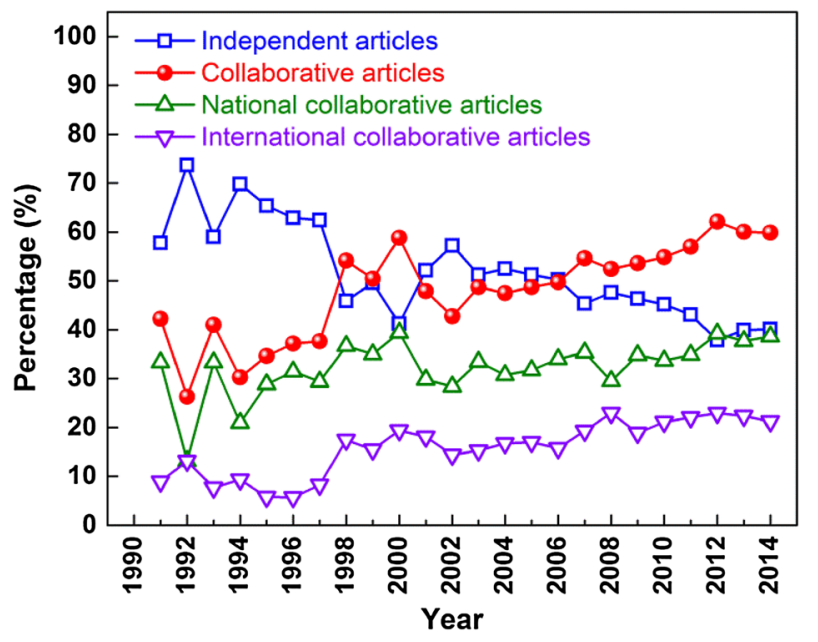

Fig. 7 Independent and collaborative share of articles per year during the research period

\section{Research tendency and hotspot}

\section{Author keywords}

Statistical analysis of author keywords, article title, article abstract, and keywords plus could be used to identify directions in science, and has proved to be efficient in comprehending and grasping the progress of new frontiers of science. Analysis of author keywords across different periods is a common bibliometric technique to analyze research trends (Hou et al. 2015; Li and Zhao 2015; Wang et al. 2015). Excluding 2566 articles without author keyword information on the ISI Web of Science, 4838 articles with author keyword information were analyzed. There were 10,724 keywords listed by authors, however, $8184(76.3 \%)$ keywords were used only once, 1225 (11.4\%) keywords were used twice, and the large number of once-only author keywords most likely indicated a lack of continuity in research and a wide disparity in research focus (Niu et al. 2014; Li and Zhao 2015). Only 205 (1.9\%) of keywords were used more than ten times, which shows that mainstream research only consistently relates to a minority of keywords. Keyword ranking changes in the four 6-year intervals are indicative of changes in research hotspots. The top 30 most frequently used keywords for the study period are listed (see in Appendix Table 5 of online supplement). Further, the co-occurrence relationships among these 30 frequently used substantives in author keywords are also visualized by a co-word network (Fig. 8).

Due to "micro/nano-bubble" being searched as title words in this study, the most frequently used author keyword of "Microbubble(s)", "Bubble(s)" and "Nanobubble(s)" should be ignored. According to the results of Appendix Table 5 of online supplement and Fig. 8, the three most frequently used keywords are "Ultrasound" (767; $15.9 \%)$, "Contrast Agent(s)" (295; $6.1 \%)$, and "Ultrasound Contrast Agent(s)" (287; $5.9 \%)$. These top 3 most frequently used substantives reflect the large amount of literature dealing with the introduction of microbubbles into a new era for personalized diagnostics, therapy, and theranostics by ultrasound contrast agents (Blomley et al. 2001; Klibanov 2006; Kiessling 


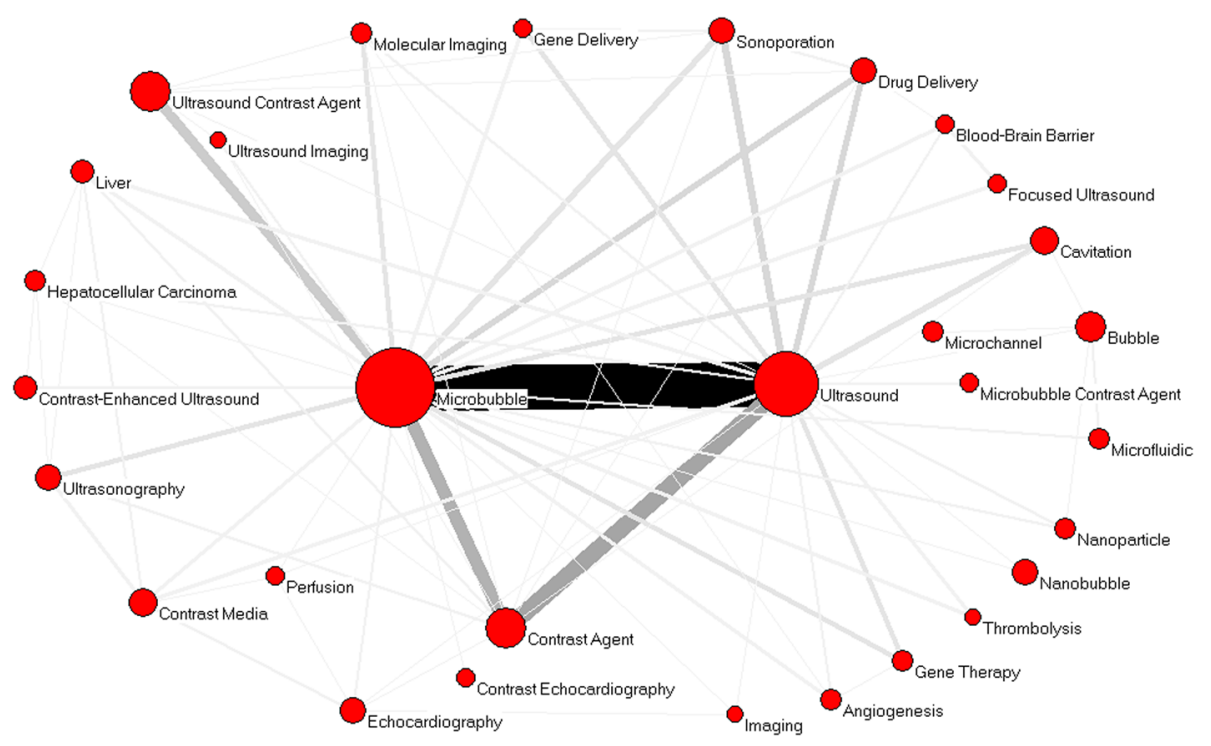

Fig. 8 Co-words network of top 30 frequently used substantives in author keywords with a minimum of eight articles

et al. 2012). "Molecular Imaging" (82; $1.7 \%$ ) did not appear in 1991-1996, while ranked 12th in 2009-2014. This could be attributed potential clinical applications of molecular imaging with microbubble-based ultrasound contrast agents that are involved in the monitoring of the biomarker status of organic tissue (Kiessling et al. 2012; Unnikrishnan and Klibanov 2012). "Drug Delivery" $(115 ; 12.4 \%)$ and "Gene Delivery" $(58 ; 1.2 \%)$ received increasing attention with a rise in rankings from no appearance in 1991-1996 to 7th and 19th in 2009-2014 respectively, showing the growing popularity of microbubbles as efficient drugs or gene carriers for therapeutic and clinical applications (Delalande et al. 2012; Sirsi and Borden 2012; Unger et al. 2014). Without targeted microbubbles as ultrasound contrast agents in the three main clinical applications including molecular imaging, drug delivery and gene delivery, their principles and methods are as follows. "Sonoporation" $(117 ; 2.4 \%)$ has an apparent upward movement in its ranks, from no emergence in 1991-1996 to 9th in 2009-2014. Further, with the introduction of microbubble-assisted ultrasound, a phenomenon called sonoporation has been successfully applied to deliver drugs and genes in vitro and in vivo in a variety of therapeutic application (Ferrara et al. 2007; Escoffre et al. 2011; Delalande et al. 2013). In addition, "Cavitation" (139; $2.9 \%)$ in author keywords steadily ranked in the top 11 during all four 6-year periods, which illustrates that ultrasound-induced microbubble cavitation has been an on-going concern (Doida et al. 1998; Postema et al. 2004; Zhang et al. 2013). Moreover, the investigation of inertial cavitation in micro-tunnels has significant implications for the development of therapeutic ultrasound applications such as ultrasound-mediated drug and gene delivery (Sassaroli and Hynynen 2007). Figure 8 shows that the relationship among "Microbubble", "Blood-Brain Barrier", and "Focused Ultrasound" was close, which the new technique of focused ultrasound with microbubble has widely applied across bloodbrain barriers for drug delivery (McDannold et al. 2012; Ting et al. 2012; Meairs 2013). Interestingly, "Echocardiography" $(117 ; 2.4 \%)$ has become routinely used diagnostic 
tests in cardiology, however, it has moved down in the rankings from 2nd in 1991-1996 to 51st in 2009-2014.

\section{Hot issues and future trends}

Analyses of research themes, trends, and hotspots can be made through finding author keywords and publication titles (Baskerville 1904). Furthermore, 'KeyWords Plus', which are different from author keywords and article title, were automatically generated by SCI from an algorithm that extracts key words from all the titles referenced or cited in the documents (Garfield 1990). A new method named "word cluster analysis" (Mao et al. 2010), which combines the author keywords, article title, and 'KeyWords Plus', has been successfully applied to trace research hotspots in previous work (Mao et al. 2015; Zheng et al. 2015b).

Research tendencies for micro/nano-bubble related research were extracted and separated into two categories of research methods including "echocardiography", "Doppler", "ultrasonography", "sonoporation", and "molecular imaging", and, research focus including "cell", "cancer", "drug", "gene", "liver", and "myocardial". In the research methods category, the "ultrasound related" were described in detail based on "ultrasound imaging", "ultrasound contrast agent", "contrast-enhanced ultrasound", and "focused ultrasound". In the research object category, "delivery related" combined the words "drug delivery", "gene delivery", "targeted delivery", "DNA delivery", "mediated delivery", and "intracellular delivery". Figure 9 shows the growth trend of hotspot-related articles during the last 24 years.

It is well known that micro/nano-bubbles have many more advantages, such as huge interfacial area, low bubble rising speed, pressurization dissolution capacity, charging surface, high mass transfer coefficient, high dissolution ability, and the release of hydroxyl radicals (Agarwal et al. 2011; Zheng et al. 2015d). Due to its unmatched superiority, micro/nano-bubbles have been widely used in various applications in many fields of science and technology. Under great pressure of increasingly new types and varieties of diseases or cancers, researchers are working to explore new detection means and novel pathways for medical applications via micro/nano-bubble. Therefore, it is crucial to analyze the dynamic development of micro/nano-bubble related research in medical applications of the past and the present. Figure 9a shows that the echocardiography and Doppler-related research have reached to a peak at the beginning of the twenty-first century, which Doppler echocardiography is a useful prediction and evaluation method in cardiology (Bax et al. 2003; Fisher et al. 2009). The research method of sonoporation first emerged in 2000 and has experienced a quickly upward movement in the last decade. This could be because the sonoporation process is a crucial key factor for the DNA, drugs and gene delivery (Delalande et al. 2013). Molecular imaging appeared in 1997 and has really come to notice since 2004, which promises to expand the range of functional imaging, enable early disease detection and therapy monitoring, and provide spatial information for imaging-guided biopsies and imaging-guided therapy (Unnikrishnan and Klibanov 2012).

Ultrasonography of particular concern in micro/nano-bubble related research include ultrasound imaging, ultrasound contrast agent, contrast-enhanced ultrasound and focused ultrasound (Staub et al. 2009; Ting et al. 2012; Unnikrishnan and Klibanov 2012). Figure $9 \mathrm{~b}$ shows that research relating to ultrasound imaging and focused ultrasound has undergone a significant increment since 2009, while ultrasound contrast agent and contrastenhanced ultrasound have increased up to the year 2011, before a period of decrement over the following 4 years. Ultrasound imaging is widely used in general tissue delineation and 
(a)
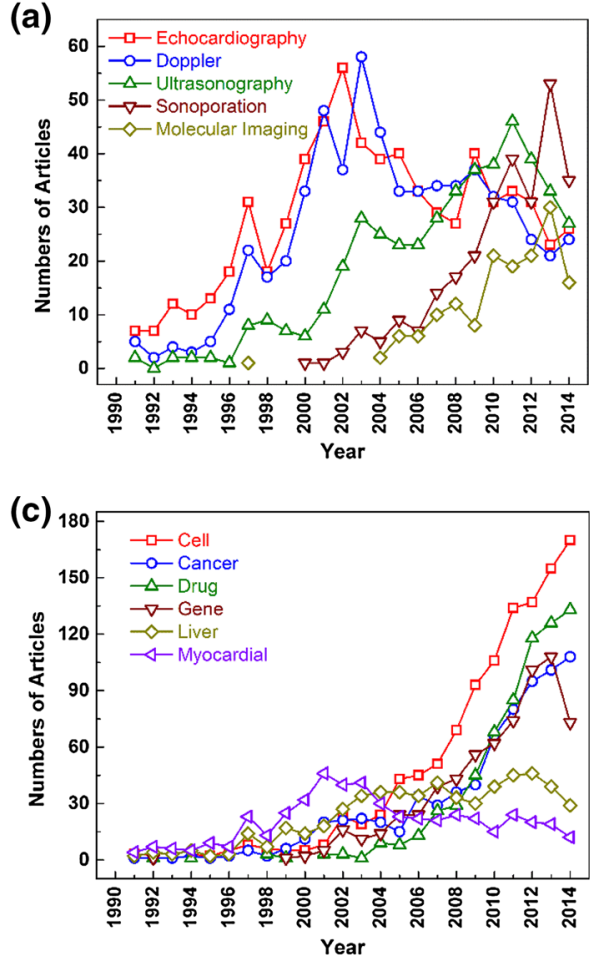

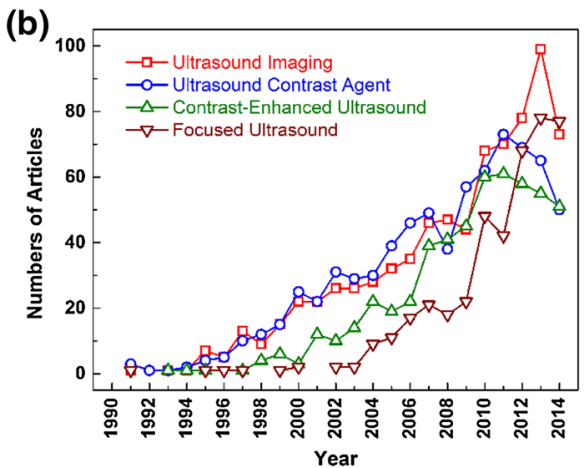

(d)

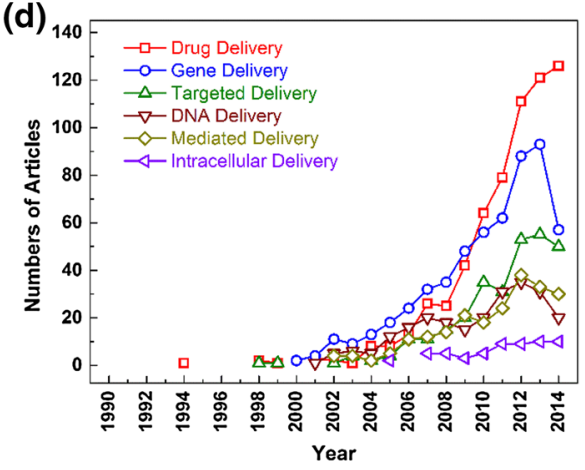

Fig. 9 Growth trends of hotpot-related articles during the last 24 years

perfusion by microbubble contrast agents (Klibanov 2006). Ultrasound contrast agents have drawn exponentially increasing interest in clinical applications and other new fields (Piscaglia et al. 2012), which its first studies on the clinical use with contrast specific imaging techniques was published at the beginning of this century (Burns et al. 2000). In comparison to ultrasound contrast agents, contrast-enhanced ultrasound has an inherent advantage, which is an opportunity to assess the contrast enhancement patterns in real time, with a much higher temporal resolution than is possible with other imaging modalities, so that the enhancement dynamics of lesions can be studied, without the need to predefine scan time points or to perform bolus tracking (Claudon et al. 2013). The focused ultrasound related research has had the highest increment rate in the recent 5 years and ranked 1st in 2014, which is extensively developed in drugs delivery, clearly showing the importance of this technique (Ting et al. 2012; Meairs 2013).

Other than research methods of research tendencies for micro/nano-bubbles, attention should be paid to the research focus due to its mission for resisting the intense pressure of increasingly new types and varieties of diseases or cancers. Figure 9c shows that cell, cancer, drug, gene, liver and myocardial related research are the most common topics in micro/nano-bubble related research (Bax et al. 2003; Ferrara et al. 2007; Escoffre et al. 2011; Delalande et al. 2013). Cell-related research has attained a tremendous increase in popularity and dominated the research focus since 2005. Cancer-related research was found in fewer than six articles in the 1990s and has had a strong growth momentum in the last decade. This could be as a result of the development of micro/nano-bubble related 
research inhibiting the cancer-related research in the initial stage and booming under modern medical conditions. Along with various kinds of diseases and cancers, drug-related research has flourished and surpassed cancer-related research since 2009. Drug-related research was in urgent demand in defeating the various illnesses caused by different pathways. Gene-related research first appeared in 1992 and has continuously developed since 1999. The drug and gene delivery applications can be realized to treat different kinds of diseases with the existence of microbubble techniques (Unger et al. 2014). Liver- and myocardial-related research has presented a stable development momentum during the last 24 years, showing that the relatively potential approaches and techniques have been used to overcome liver- and myocardial-related issues (Narsinh et al. 2011; Lomonaco et al. 2013).

In recent years, the other one research focus of micro/nano-bubbles was their role as efficient carriers in object delivery including drug, gene and DNA delivery, and delivery pathways including targeted, mediated and intracellular delivery. Figure 9d shows that the drug delivery is the first delivery method with microbubble technique in Suslick et al. (1994) and has a rocketing development since 2008. Gene delivery also has a significant increment in the last decade but an unexpected decline in 2014. The related research of drug and gene delivery was found more than 550 articles and was the top 2 hotspots in object delivery related research. The other four object delivery related research followed distantly with less than 300 articles. The results reveal that increasingly popular of drug and gene delivery during the therapeutic and clinical applications field of microbubble ultrasound contrast agents (shown in Fig. 8) (Ferrara et al. 2007). DNA delivery can be observed in the areas of the body where ultrasound is applied after intravascular administration of microbubbles and plasmid DNA (Hernot and Klibanov 2008). In the case of delivery pathways, targeted delivery, combing with microbubble targeting and ultrasoundassisted microbubble-based drug-delivery systems, was always used in the application of targeted personalized diagnostics and therapy (Klibanov 2006). Mediated delivery was one kind of assistant measurements for supporting the ultrasound gene or drug transfer process (Koike et al. 2005; Wang et al. 2008). Intracellular delivery had been extensively applied in short interfering RNA (siRNA) or drug delivery by microbubble-enhanced ultrasound (Kinoshita and Hynynen 2005a, 2005b; Dixon et al. 2013). Nevertheless, the object delivery and delivery pathways are close interaction with each other and aim at eliminating cancer or illness.

\section{Conclusions}

Based on 7404 micro/nano-bubble related articles from SCI-EXPANDED, this bibliometric study provided an overview of research in micro/nano-bubble related research and identified some significant points in this field throughout the investigation period. The amount of literature dedicated to micro/nano-bubble related research has significantly increased over the last 20 years. This field showed exponential growth in cumulative number of publications from the year 1991, which implies that the cumulative number of articles in 2019 will be twice than of 2014. Articles were published in 1476 journals across 103 subject categories. Ultrasound in Medicine and Biology with the highest h-index of 56 published the most $(6.9 \%)$ articles in this, followed by Langmuir and Journal of the Acoustical Society of America. The researchers mainly focused on the categories of 'Radiology, Nuclear Medicine \& Medical Imaging' and 'Engineering'. USA and Univ 
Toronto, Canada were the most productive country and institution, respectively, while the USA, with the most international collaborative articles and highest $h$-index of 111 , was the major collaborator with other 20 countries in the top 25 most productive countries. The G7 accounted for $59.4 \%$ of total world production. Word cluster analysis was successfully used to trace popular micro/nano-bubble related research hot topics, which showed that the core of micro/nano-bubble related research is in the progress of medical applications in response to efficient, accurate and complex medical requirements. The most common micro/nano-bubble related research in medical applications were methods such as echocardiography, Doppler, ultrasonography, sonoporation and molecular imaging, which have attracted much concern with ever-increasing demand by medical conditions and human health, and can be considered popular research topics in recent years. The most common research focus of micro/nano-bubbles was their role as efficient carriers in object delivery including drug, gene and DNA delivery, and delivery pathways including targeted, mediated and intracellular delivery, all of which drew great attention in recent decades due to severe challenges and tests for resisting the intense pressure of increasingly new types and varieties of diseases or cancers.

Acknowledgments The authors gratefully acknowledge the financial support of the Major Science and Technology Program for Water Pollution Control and Treatment (2012ZX07201002-6).

\section{References}

Agarwal, A., Ng, W. J., \& Liu, Y. (2011). Principle and applications of microbubble and nanobubble technology for water treatment. Chemosphere, 84(9), 1175-1180.

Aoi, A., Watanabe, Y., Mori, S., Takahashi, M., Vassaux, G., \& Kodama, T. (2008). Herpes simplex virus thymidine kinase-mediated suicide gene therapy using nano/microbubbles and ultrasound. Ultrasound in Medicine and Biol, 34(3), 425-434.

Baskerville, C. (1904). The titles of papers. Science (New York, NY), 19(487), 702-703.

Bax, J. J., Molhoek, S. G., van Erven, L., Voogd, P. J., Somer, S., Boersma, E., et al. (2003). Usefulness of myocardial tissue Doppler echocardiography to evaluate left ventricular dyssynchrony before and after biventricular pacing in patients with idiopathic dilated cardiomyopathy. The American Journal of Cardiology, 91(1), 94-97.

Bell, M., \& Pavitt, K. (1997). Technological accumulation and industrial growth: Contrasts between developed and developing countries. Cambridge: Cambridge University Press.

Blomley, M. J. K., Cooke, J. C., Unger, E. C., Monaghan, M. J., \& Cosgrove, D. O. (2001). Microbubble contrast agents: A new era in ultrasound. BMJ. British Medical Journal, 322(7296), 1222-1225.

Burns, P. N., Wilson, S. R., \& Simpson, D. H. (2000). Pulse inversion imaging of liver blood flow: Improved method for characterizing focal masses with microbubble contrast. Investigative Radiology, 35(1), 58.

Ciriminna, R., \& Pagliaro, M. (2013). On the use of the $h$-index in evaluating chemical research. Chemistry Central Journal, 7(1), 132.

Claudon, M., Dietrich, C. F., Choi, B. I., Cosgrove, D. O., Kudo, M., Nolsøe, C. P., et al. (2013). Guidelines and good clinical practice recommendations for contrast enhanced ultrasound (CEUS) in the liverupdate 2012. Ultraschall in der Medizin, 34(1), 11-29.

De Nooy, W., Mrvar, A., \& Batagelj, V. (2011). Exploratory social network analysis with Pajek. Cambridge: Cambridge University Press.

Delalande, A., Kotopoulis, S., Postema, M., Midoux, P., \& Pichon, C. (2013). Sonoporation: Mechanistic insights and ongoing challenges for gene transfer. Gene, 525(2), 191-199.

Delalande, A., Postema, M., Mignet, N., Midoux, P., \& Pichon, C. (2012). Ultrasound and microbubbleassisted gene delivery: Recent advances and ongoing challenges. Therapeutic Delivery, 3(10), 1199-1215.

Dixon, A. J., Dhanaliwala, A. H., Chen, J. L., \& Hossack, J. A. (2013). Enhanced intracellular delivery of a model drug using microbubbles produced by a microfluidic device. Ultrasound in Medicine and Biology, 39(7), 1267-1276. 
Doida, Y., Marcello, K. R., Brayman, A. A., Cox, C., Barned, S., \& Miller, M. W. (1998). Sonochemicals increase the mutation frequency of V79 cells in vitro. Ultrasound in Medicine and Biology, 24(8), 1209-1213.

Endo, A., Srithongouthai, S., Nashiki, H., Teshiba, I., Iwasaki, T., Hama, D., \& Tsutsumi, H. (2008). DOincreasing effects of a microscopic bubble generating system in a fish farm. Marine Pollution Bulletin, 57(1), 78-85.

Escoffre, J. M., Piron, J., Novell, A., \& Bouakaz, A. (2011). Doxorubicin delivery into tumor cells with ultrasound and microbubbles. Molecular Pharmaceutics, 8(3), 799-806.

Ferrara, K., Pollard, R., \& Borden, M. (2007). Ultrasound microbubble contrast agents: Fundamentals and application to gene and drug delivery. Annual Review of Biomedical Engineering, 9, 415-447.

Finardi, U. (2015). Scientific collaboration between BRICS countries. Scientometrics, 102(2), 1139-1166.

Fisher, M. R., Forfia, P. R., Chamera, E., Housten-Harris, T., Champion, H. C., Girgis, R. E., et al. (2009). Accuracy of Doppler echocardiography in the hemodynamic assessment of pulmonary hypertension. American Journal of Respiratory and Critical Care Medicine, 179(7), 615-621.

Fu, H. Z., Long, X., \& Ho, Y. S. (2014). China's research in chemical engineering journals in Science Citation Index Expanded: A bibliometric analysis. Scientometrics, 98(1), 119-136.

Gao, W., Chen, Y., Liu, Y., \& Guo, H. (2015). Scientometric analysis of phosphorus research in eutrophic lakes. Scientometrics, 102(3), 1951-1964.

Gao, Z., Kennedy, A. M., Christensen, D. A., \& Rapoport, N. Y. (2008). Drug-loaded nano/microbubbles for combining ultrasonography and targeted chemotherapy. Ultrasonics, 48(4), 260-270.

Garfield, E. (1990). Key-words-plus takes you beyond title words. 2. Expanded journal coverage for currentcontents-on-diskette includes social and behavioral-sciences. Current Contents, 33, 5-9.

Hernot, S., \& Klibanov, A. L. (2008). Microbubbles in ultrasound-triggered drug and gene delivery. Advanced Drug Delivery Reviews, 60(10), 1153-1166.

Hirsch, J. E. (2005). An index to quantify an individual's scientific research output. Proceedings of the National Academy of Sciences of the United States of America, 102(46), 16569-16572.

Hirsch, J. E. (2007). Does the h index have predictive power? Proceedings of the National Academy of Sciences, 104(49), 19193-19198.

Hou, Q., Mao, G., Zhao, L., Du, H., \& Zuo, J. (2015). Mapping the scientific research on life cycle assessment: A bibliometric analysis. The International Journal of Life Cycle Assessment, 20(4), 541-555.

Invernizzi, N., Foladori, G., Robles-Belmont, E., Lau, E. Z., Figueroa, E. A., Bagattolli, C., et al. (2015). Nanotechnology for social needs: Contributions from Latin American research in the areas of health, energy and water. Journal of Nanoparticle Research, 17(5), 1-19.

Jiang, L., Wong, M., \& Zohar, Y. (1999). Phase change in microchannel heat sinks with integrated temperature sensors. Journal of Microelectromechanical Systems, 8(4), 358-365.

Karpagam, R. (2014). Global research output of nanobiotechnology research: A scientometrics study. Current Science, 106(11), 1490.

Kiessling, F., Fokong, S., Koczera, P., Lederle, W., \& Lammers, T. (2012). Ultrasound microbubbles for molecular diagnosis, therapy, and theranostics. Journal of Nuclear Medicine, 53(3), 345-348.

Kinoshita, M., \& Hynynen, K. (2005a). A novel method for the intracellular delivery of siRNA using microbubble-enhanced focused ultrasound. Biochemical and Biophysical Research Communications, 335(2), 393-399.

Kinoshita, M., \& Hynynen, K. (2005b). Intracellular delivery of Bak BH3 peptide by microbubble-enhanced ultrasound. Pharmaceutical Research, 22(5), 716-720.

Klibanov, A. L. (2006). Microbubble contrast agents: Targeted ultrasound imaging and ultrasound-assisted drug-delivery applications. Investigative Radiology, 41(3), 354-362.

Koike, H., Tomita, N., Azuma, H., Taniyama, Y., Yamasaki, K., Kunugiza, Y., et al. (2005). An efficient gene transfer method mediated by ultrasound and microbubbles into the kidney. The Journal of Gene Medicine, 7(1), 108-116.

Lee, M., Lee, E. Y., Lee, D., \& Park, B. J. (2015). Stabilization and fabrication of microbubbles: Applications for medical purposes and functional materials. Soft Matter, 11(11), 2067-2079.

Levy, N., Burke, S. A., Meaker, K. L., Panlasigui, M., Zettl, A., Guinea, F., et al. (2010). Strain-induced pseudo-magnetic fields greater than 300 tesla in graphene nanobubbles. Science, 329(5991), 544-547.

Li, P., \& Tsuge, H. (2006). Water treatment by induced air flotation using microbubbles. Journal of Chemical Engineering of Japan, 39(8), 896-903.

Li, W., \& Zhao, Y. (2015). Bibliometric analysis of global environmental assessment research in a 20-year period. Environmental Impact Assessment Review, 50, 158-166.

Lomonaco, R., Sunny, N. E., Bril, F., \& Cusi, K. (2013). Nonalcoholic fatty liver disease: Current issues and novel treatment approaches. Drugs, 73(1), 1-14. 
Mao, G., Liu, X., Du, H., Zuo, J., \& Wang, L. (2015). Way forward for alternative energy research: A bibliometric analysis during 1994-2013. Renewable and Sustainable Energy Reviews, 48, 276-286.

Mao, N., Wang, M., \& Ho, Y. (2010). A bibliometric study of the trend in articles related to risk assessment published in Science Citation Index. Human and Ecological Risk Assessment, 16(4), 801-824.

McDannold, N., Arvanitis, C. D., Vykhodtseva, N., \& Livingstone, M. S. (2012). Temporary disruption of the blood-brain barrier by use of ultrasound and microbubbles: Safety and efficacy evaluation in rhesus macaques. Cancer Research, 72(14), 3652-3663.

Meairs, S. (2013). Drug delivery across the blood-brain barrier with focused ultrasound and microbubbles. In The Blood Brain Barrier (BBB) (pp. 143-158). Berlin/Heidelberg: Springer.

Narsinh, K., Narsinh, K. H., \& Wu, J. C. (2011). Derivation of human induced pluripotent stem cells for cardiovascular disease modeling. Circulation Research, 108(9), 1146-1156.

Neethirajan, S., Kobayashi, I., Nakajima, M., Wu, D., Nandagopal, S., \& Lin, F. (2011). Microfluidics for food, agriculture and biosystems industries. Lab on a Chip, 11(9), 1574-1586.

Niu, B. B., Hong, S., Yuan, J. F., Peng, S., Wang, Z., \& Zhang, X. (2014). Global trends in sediment-related research in earth science during 1992-2011: A bibliometric analysis. Scientometrics, 98(1), 511-529.

Persson, O. (1994). The intellectual base and research fronts of JASIS 1986-1990. Journal of the American Society for Information Science, 45(1), 31-38.

Persson, O., Danell, R., \& Schneider, J. W. (2009). How to use Bibexcel for various types of bibliometric analysis. In Celebrating scholarly communication studies: A Festschrift for Olle Persson at his 60th birthday (pp. 9-24).

Piscaglia, F., Nolsøe, C., Dietrich, C. A., Cosgrove, D. O., Gilja, O. H., Bachmann Nielsen, M., et al. (2012). The EFSUMB guidelines and recommendations on the clinical practice of contrast enhanced ultrasound (CEUS): Update 2011 on non-hepatic applications. Ultraschall in der Medizin, 33(1), 33.

Postema, M., Van Wamel, A., Lancée, C. T., \& De Jong, N. (2004). Ultrasound-induced encapsulated microbubble phenomena. Ultrasound in Medicine and Biology, 30(6), 827-840.

Pritchard, A. (1969). Statistical bibliography or bibliometrics. Journal of Documentation, 25(4), 348-349.

Rodríguez-Rodríguez, J., Sevilla, A., Martínez-Bazán, C., \& Gordillo, J. M. (2015). Generation of microbubbles with applications to industry and medicine. Annual Review of Fluid Mechanics, 47, $405-429$.

Sassaroli, E., \& Hynynen, K. (2007). Cavitation threshold of microbubbles in gel tunnels by focused ultrasound. Ultrasound in Medicine and Biology, 33(10), 1651-1660.

Sato, K. (2011). Recent patents on micro-and nano-bubble applications and potential application of a swirltype generator. Recent Patents on Mechanical Engineering, 4(3), 202-211.

Sebba, F. (1971). Microfoams-an unexploited colloid system. Journal of Colloid and Interface Science, 35(4), 643-646.

Sirsi, S. R., \& Borden, M. A. (2012). Advances in ultrasound mediated gene therapy using microbubble contrast agents. Theranostics, 2(12), 1208.

Staub, F., Tournoux-Facon, C., Roumy, J., Chaigneau, C., Morichaut-Beauchant, M., Levillain, P., et al. (2009). Liver fibrosis staging with contrast-enhanced ultrasonography: Prospective multicenter study compared with METAVIR scoring. European Radiology, 19(8), 1991-1997.

Suslick, K. S., Grinstaff, M. W., Kolbeck, K. J., \& Wong, M. (1994). Characterization of sonochemically prepared proteinaceous microspheres. Ultrasonics Sonochemistry, 1(1), S65-S68.

Terekhov, A. I. (2015). R\&D on carbon nanostructures in Russia: scientometric analysis, 1990-2011. Journal of Nanoparticle Research, 17(2), 1-26.

Thomson Reuters. (2015). Quartiles in JCR on the InCites platform. http://ipscience-help.thomsonreuters. com/incitesLiveJCR/JCRGroup/jcrJournalProfile/jcrJournalProfileRank.html.

Ting, C., Fan, C., Liu, H., Huang, C., Hsieh, H., Yen, T., et al. (2012). Concurrent blood-brain barrier opening and local drug delivery using drug-carrying microbubbles and focused ultrasound for brain glioma treatment. Biomaterials, 33(2), 704-712.

Unger, E., Porter, T., Lindner, J., \& Grayburn, P. (2014). Cardiovascular drug delivery with ultrasound and microbubbles. Advanced Drug Delivery Reviews, 72, 110-126.

Unnikrishnan, S., \& Klibanov, A. L. (2012). Microbubbles as ultrasound contrast agents for molecular imaging: Preparation and application. American Journal of Roentgenology, 199(2), 292-299.

Wang, M., Liu, D., Jia, J., \& Zhang, X. (2015). Global trends in soil monitoring research from 1999-2013: A bibliometric analysis. Acta Agriculturae Scandinavica, Section B-Soil and Plant Science, 65(6), 483-495.

Wang, J., Wang, J., Chen, H., Zhang, C., Liu, L., Pan, S., \& Wu, C. (2008). Ultrasound-mediated microbubble destruction enhances gene transfection in pancreatic cancer cells. Advances in Therapy, 25(5), 412-421. 
Wang, L., Wang, Q., Zhang, X., Cai, W., \& Sun, X. (2013). A bibliometric analysis of anaerobic digestion for methane research during the period 1994-2011. Journal of Material Cycles and Waste Management, 15(1), 1-8.

Wei, K., Jayaweera, A. R., Firoozan, S., Linka, A., Skyba, D. M., \& Kaul, S. (1998). Quantification of myocardial blood flow with ultrasound-induced destruction of microbubbles administered as a constant venous infusion. Circulation, 97(5), 473-483.

Xu, J. L., Cheng, P., \& Zhao, T. S. (1999). Gas-liquid two-phase flow regimes in rectangular channels with mini/micro gaps. International Journal of Multiphase Flow, 25(3), 411-432.

Xu, Q., Nakajima, M., Ichikawa, S., Nakamura, N., \& Shiina, T. (2008). A comparative study of microbubble generation by mechanical agitation and sonication. Innovative Food Science and Emerging Technologies, 9(4), 489-494.

Xu, Q., Nakajima, M., Liu, Z., \& Shiina, T. (2011). Biosurfactants for microbubble preparation and application. International Journal of Molecular Sciences, 12(1), 462-475.

Yu, Q., Shao, H., \& Duan, Z. (2011). Research groups of oncology co-authorship network in China. Scientometrics, 89(2), 553-567.

Zhai, L., Pan, Y., Guo, Y., Ma, Z., \& Bi, F. (2014). International comparative study on nanofiltration membrane technology based on relevant publications and patents. Scientometrics, 101(2), 1361-1374.

Zhang, C., Cao, H., Li, Q., Tu, J., Guo, X., Liu, Z., \& Zhang, D. (2013). Enhancement effect of ultrasoundinduced microbubble cavitation on branched polyethylenimine-mediated VEGF165 transfection with varied N/P ratio. Ultrasound in Medicine and Biology, 39(1), 161-171.

Zheng, T., Wang, J., Wang, Q., Meng, H., \& Wang, L. (2015a). Research trends in electrochemical technology for water and wastewater treatment. Applied Water Science,. doi:10.1007/s13201-0150280-4.

Zheng, T., Wang, J., Wang, Q., Nie, C., Smale, N., Shi, Z., \& Wang, X. (2015b). A bibliometric analysis of industrial wastewater research: current trends and future prospects. Scientometrics, 105(2), 863-882.

Zheng, T., Wang, Q., Shi, Z., Huang, P., Li, J., Zhang, J., \& Wang, J. (2015c). Separation of pollutants from oil-containing restaurant wastewater by novel microbubble air flotation and traditional dissolved air flotation. Separation Science and Technology, 50(16), 2568-2577.

Zheng, T., Wang, Q., Zhang, T., Shi, Z., Tian, Y., Shi, S., et al. (2015d). Microbubble enhanced ozonation process for advanced treatment of wastewater produced in acrylic fiber manufacturing industry. Journal of Hazardous Materials, 287, 412-420.

Zhou, P., \& Leydesdorff, L. (2006). The emergence of China as a leading nation in science. Research Policy, 35(1), 83-104. 